\title{
APROXIMACIONES PSICOLÓGICAS AL TRATAMIENTO DE LAS ESQUIZOFRENIAS
}

\author{
ENCARNACIÓN SANTIAGO, ALMA D. MARTÍNEZ DE SALAZAR, JUAN C. IBÁÑEZ \\ Y M. JOSÉ SÁNCHEZ \\ Complejo Hospitalario Torrecárdenas, Almería
}

(Recibido el 17 de noviembre de 1998)

\begin{abstract}
Los pacientes que sufren cualquier tipo de esquizofrenia presentan alteraciones importantes en sus funciones mentales superiores así como dificultades conductuales y sociales significativas.

Desde distintos enfoques teóricos se ha intentado analizar dichas dificultades a la vez que encontrar la manera de orientar el abordaje terapéutico de estos pacientes.

En este trabajo hemos repasado las aportaciones que, desde distintos modelos te6ricos del ámbito de la Psicologia, han supuesto un avance en las posibilidades terapéuticas de los sujetos afectos de esquizofrenia y, por tanto, una mejoría en su calidad de vida y en su integración social., siendo especialmente importante la exitencia de planes terapéuticos integradores como por ejemplo el modelo propuesto por Roder, Brenner, Hodel y Kienzle, (1996).
\end{abstract}

Palabras clave: Esquizofrenia, tratamiento psicológico.

\section{Approach of psychological treatment of schizophrenia}

TPatients with schizophrenia have some important failures in their superior mental functions and significative behavioral and social difficulties.

Those difficulties have been analyzed from several theoretical frames. At the same time, therapeutic posibilities for these patients have been taken into account in order to find them out.

In this work we have revised the detections that, from differents psychological theories, have become an advance in the treatment alternatives for persons who suffer from schizophrenia and, so, an improvement in their lives and social integration. We have wanted to show the importance of an integral model like it has been proposed by Roder, Brenner, Hodel and Kienzle, 1996.

Key words: Schizophrenia, psychological treatment.

\section{EL ABORDAJE PSICOLÓGICO DE LAS ESQUIZOFRENIAS}

\section{INTRODUCCIÓN}

La esquizofrenia es un trastorno heterogéneo en sus síntomas, curso y forma clínica que afecta a las principales funciones mentales y tiene unas repercusiones

Correspondencia: Alma D. Martínez de Salazar, c/ Plaza Circular n ${ }^{2} 13,8 .{ }^{2} \mathrm{C}, 30008$ Murcia. Tfno: 968 204 870. alma@correo.cop.es. conductuales y sociales importantes. Es uno de los trastornos mentales más graves que existen. Su costo económico, social y humano es elevado, necesitándose importantes recursos para realizar un tratamiento integral del paciente esquizofrénico.

Los factores etiológicos de la esquizofrenia no están totalmente definidos. Los modelos tradicionales que tratan de dar explicación de las causas de este trastorno se han movido en un continuo que va desde el polo biológico-molecular hasta la 
teoría de campo, pasando por modelos intermedios como el neurofisiológico, el evolutivo y el de la Teoría del Aprendizaje. Ningún modelo ha podido ofrecer una explicación etiológica definitiva de la esquizofrenia y en la actualidad lo más aceptado es una aproximación explicativa al trastorno desde modelos integradores como el Modelo de la Vulnerabilidad propuesto por Zubin y Spring $(1977,1978)$.

Los signos y síntomas de la esquizofrenia ya aparecen descritos en escritos de varios milenios antes de Cristo, siendo en el siglo XIX y comienzos del XX cuando se realiza una aproximación más fina a su descripción y clasificación diferenciando distintos subtipos. Se atribuye a dos psiquiatras alemanes, Kraepelin y Bleuler, la aproximación científica al concepto central de la esquizofrenia. Kraepelin fue el primero en identificar el fenómeno agrupando unas entidades ya conocidas: la psicosis paranoide descrita por W. Sander en 1868 , la hebefrenia propuesta por E. Hecker en 1871 y la catatonía descrita por K.L. Kahlbaum en 1874 (datos citados por Carpenter y Stephens, 1979). Emil Kraepelin, en su Compendio de Psiquiatría de 1871, consideró las psicosis paranoides, la hebefrénica y la catatonía como enfermedades separadas. Sin embargo, unos años después agrupó estas tres enfermedades y habló de una sola entidad mórbida, de comienzo temprano y progresivo deterioro intelectual: la demencia praecox. Posteriormente, Bleuler en su monografía Demencia Precoz o el grupo de las esquizofrenias (1911), introdujo el concepto de esquizofrenia y criticó algunos de los conceptos de Kraepelin (en concreto, el énfasis en la demencia, el comienzo precoz y el pronóstico desfavorable), manteniendo la existencia de un grupo de psicosis de etiología heterogénea. Bleuler mantuvo los tres subtipos kraepelianos de demencia precoz: paranoia, hebefrenia y catatonía, y añadió el de esquizofrenia simple.
La clasificación propuesta por estos autores ha sido considerada como los subtipos clásicos de la esquizofrenia. Sin embargo el descontesto con dicha clasificación clásica ha ido en aumento con el tiempo. La heterogeneidad de la esquizofrenia es una realidad asumida por los clínicos y los investigadores. Los pacientes tienen con mucha frecuencia síntomas mixtos o "pasan» de un subtipo a otro. El propio diagnóstico de la esquizofrenia como los subtipos clásicos tiene una fiabilidad y validez demasiado bajas. Esta insatisfacción ha llevado a la búsqueda de nuevos enfoques que redujeran la variabilidad grupal y respondieran a las muestras de clasificación en la clínica e investigación. En este sentido se han propuesto las siguientes dimensiones dominantes en la investigación de la esquizofrenia: «agudo-crónico», "paranoide-no paranoide» y "procesal-reactivo". La tipología propuesta por Crow (1980), trata de relacionar la psicopatología con las alteraciones biológicas que se van descubriendo del trastorno y divide los síntomas de la esquizofrenia en positivos y negativos. Precisamente, la clasificación en función de los síntomas ha sido cada vez más utilizada (Frith, 1992; Andreansen, 1985). Los síntomas positivos se refieren a aquellos síntomas de distorsión o exageración de las funciones psicológicas, mientras que los negativos se refieren a síntomas de pérdida o disminución de funciones.

El tratamiento de los pacientes esquizofrénicos ha sido muy diferente a lo largo del tiempo y se ha configurado conforme al modelo teórico explicativo de partida. Las intervenciones han abarcado la muerte en la hoguera, los «banquillos de inmersiones», enemas diarios, extracción de toda la dentadura, la ludoterapia etc. Ya en el siglo XX, entre las intervenciones empleadas aparecen el electrochoque, el coma insulínico, la neurocirugía y el aislamiento. Es a partir de 
mediados de este siglo cuando la aparición de los neurolépticos abrió nuevas posibilidades de intervención del paciente en la comunidad. No cabe duda que un avance en el tratamiento de la esquizofrenia vino de la mano del descubrimiento de los neurolépticos. Su capacidad de controlar, aunque parcialmente, la sintomatología psicótica positiva permitió externalizar a los pacientes esquizofrénicos de las instituciones cerradas y llevar a cabo otras intervenciones psicosociales complementarias. Sin embargo estos fármacos tienen sus limitaciones. Un número considerable de pacientes tratados sigue experimentando delirios y alucinaciones; y hasta un $30-40 \%$ de los pacientes presentarán recaídas durante el tratamiento farmacológico (Johnson, Ludlow, Street y Taylor, 1987). Además los fármacos neurolépticos producen una serie de efectos secundarios indeseables que favorecen el incumplimiento de las prescripciones en un grupo significativo de enfermos. Precisamente por las limitaciones de la farmacoterapia y de los tratamientos psiquiátricos tradicionales en la esquizofrenia se justifica la necesidad de llevar a cabo otros abordajes terapéuticos.

Este trabajo pretende describir algunas de las diferentes aproximaciones que desde el ámbito de la Psicología se han venido realizando en el tratamiento de los pacientes con síntomas esquizofrénicos. El abordaje psicológico de estos pacientes ha contemplado numerosas áreas de intervención (dirigidas al control de los delirios y alucinaciones, a favorecer conductas de autonomía y sociabilidad, a establecer una buena adherencia al tratamiento etc.) y a distintos niveles (intervenciones individuales, grupales y familiares). Dicha variedad en las intervenciones psicológicas en el tratamiento del paciente esquizofrénico dificulta su clasificación; a pesar de ello, con objeto de facilitar la exposición del tema que nos ocupa, las hemos ordenado en los siguientes apartados:

- Intervenciones dirigidas al control de la sintomatología negativa

- Intervenciones dirigidas al control de la sintomatología positiva

- Intervenciones dirigidas a la prevención de recaídas:

- Programas de intervención precoz

- Programas de intervención con familias

- Intervenciones dirigidas a potenciar los recursos personales del paciente.

- Intervenciones dirigidas a la rehabilitación de funciones cognitivas

\section{INTERVENCIONES DIRIGIDAS AL CONTROL DE LOS SÍNTOMAS NEGATIVOS}

La clínica negativa de los pacientes esquizofrénicos hace referencia a una pérdida o disminución de las funciones psicológicas que se manifiesta en una reducción del comportamiento espontáneo, empobrecimiento del lenguaje y de la capacidad ideativa y retraimiento social. Andreasen (1985) señalaba como rasgos negativos asociados con la esquizofrenia el aplanamiento o embotamiento de la afectividad (expresión facial invariable, disminución de los movimientos espontáneos, ausencia de gestos expresivos y de inflexiones vocales), la alogia (pobreza del habla y de su contenido, aumento de la latencia de la respuesta), la avolición-apatía (poco cuidado personal e higiene, falta de energía y persistencia en el trabajo) y la anhedoniaasociabilidad (pérdida de interés en la diversión y el sexo, incapacidad para sentir intimidad y crear amigos).

Los signos negativos que presentan los pacientes esquizofrénicos se han abordado con estrategias psicológicas diferentes. 
En la década de los sesenta numerosas intervenciones desde el paradigma conductual operante con pacientes esquizofrénicos crónicos institucionalizados se dirigían a modificar la clínica negativa (Stahl y Leitenberg, 1982). El Modelo Conductual, basado en la Teoría del Aprendizaje y en las técnicas que se derivan de ésta, tiene como planteamiento básico que la conducta se aprende y fortalece como resultado de sus consecuencias. Desde este enfoque el objetivo de la intervención consiste en relacionar las conductas inadaptadas con otros sucesos observables y, a través de las técnicas de Modificación de Conducta, sustituirlas por otras más adaptadas.

En la revisión que realizan Stahl y Leitenberg (1982) sobre el tratamiento psicológico en pacientes esquizofrénicos crónicos señalan la técnica de Economía de Fichas y los Programas de Reforzamiento individualizados como las más utilizadas con los pacientes que se encuentran en instituciones.

La economía de fichas es una técnica basada en el control de estímulos a través del refuerzo condicionado. El paciente puede conseguir fichas (refuerzo condicionado) que puede canjear por otros reforzadores (actividades, privilegios y comodidades adecuadas al ámbito hospitalario en cuestión), contingentes a las conductas objetivo. Existe la posibilidad de sanciones (retirada de fichas) por la aparición de conductas no deseadas.

La mayoría de los estudios de economía de fichas con pacientes mentales crónicos han sido orientados, principalmente, a instaurar conductas saludables (cuidado personal, de interacción social, participación en actividades grupales, implicación en el trabajo etc.) y a controlar conductas altamente disruptivas o violentas. Los reforzadores han sido variados y adecuados al ámbito hospitalario en el que se aplican (acceso a privilegios materiales o sociales, realización de activida- des, comodidades etc.) lo que también ha dificultado su posterior generalización.

Son numerosos los estudios dirigidos al control de la clínica negativa en pacientes esquizofrénicos que utilizaron esta técnica en la década de los 60 . Ayllon y Azrin (1968) incrementaron el nivel de ejecución de pacientes psiquiátricos crónicos institucionalizados con un programa de economía de fichas, independientemente de la edad, el nivel de educación y el tiempo de hospitalización. Lloyd y Garlington (1968) incrementaron con esta misma técnica la conducta de cuidado personal (definida por la acción de peinarse, maquillarse, higiene personal, limpieza de la ropa etc.) en 13 pacientes esquizofrénicas crónicas. Otros signos sobre los que se ha intervenido han sido la conducta de apatía. Schaefer y Martin (1966) trataron con un programa de economía de fichas la conducta apática de 40 mujeres pacientes mentales crónicas. Estos autores definieron «la conducta apática» como aquel comportamiento mutuo excluyente (andar, sentarse, acostarse) sin la ejecución simultanea de otra conducta concomitante (hablar, leer, escuchar). Diferenciaron un grupo control de un grupo experimental. Los resultados orientan a que mientras que en el grupo experimental se observó un marcado descenso en la conducta apática, los pacientes que pertenecían al grupo control solo obtuvieron un ligero descenso en ésta. Atthowe y Krasner (1968) también trabajaron sobre la conducta de apatía con 86 pacientes mentales crónicos hospitalizados. Definieron dicha conducta en función del nivel de actividad, responsabilidad, planificación de futuro, decisiones responsables y actividad rutinaria adaptativas. Su programa de intervención consistio en un programa de economía de fichas con periodo previo de moldeamiento al empleo de las fichas y refuerzo social asociado a la consecución de éstas. Los 
resultados mostraron un incremento de la responsabilidad y autosuficiencia, disminución de la apatía, incremento de la actividad y generalización a otras conductas adaptadas.

También se ha abordado la clínica negativa de los pacientes esquizofrénicos con programas de corte operante individualizados; utilizándose técnicas como el refuerzo positivo y negativo, la extinción, el moldeamiento, el control de contingencias y el tiempo fuera.

Las conductas objetivo que se han modificado con los programas individualizados han sido variadas. Mertins y Fuller (1963) incrementaron la frecuencia de afeitado en pacientes psicóticos regresivos a través de reforzamiento positivo y moldeamiento. Hilby, Stenmerk y Horner (1967) favorecieron la conducta de autotraslado en un paciente psicótico confinado a una silla de ruedas con reforzamiento social. Agras (1967) regularizó las ingestas alimentarias de un paciente esquizofrénico que había dejado de comer regularmente tres años antes, utilizando la retirada de atención a sus negativas a comer y el control estimular. Moore y Crom (1969) trataron a una paciente esquizofrénica crónica con obesidad por exceso de ingesta de alimentos con un programa de contingencias discriminativas al aumento o descenso de peso.

En definitiva, estos estudios y otros tantos vienen a demostrar que en los pacientes esquizofrénicos, como en cualquier otra persona, son modificables conductas a través de las técnicas que nos provee la Terapia de Conducta como venimos comprobando en nuestra práctica clínica.

Otra importante área de abordaje de la clínica negativa que presentan los pacientes esquizofrénicos es realizada a través de los Programas de Entrenamiento en Habilidades Sociales (E.H.S.).

La capacitación social incluye un amplio repertorio de habilidades que per- miten a las personas afrontar eficazmente situaciones específicas. El entrenamiento en habilidades sociales se ha consolidado como uno de los componentes psicosociales más importantes de un programa de tratamiento integral para pacientes con esquizofrenia (Bellack y Mneser, 1993). Estos programas se han basado en la idea de que los déficits en conductas sociales simples de los pacientes esquizofrénicos subyacen a problemas interpersonales más generales como el aislamiento o la falta de asertividad.

Durante el episodio psicótico agudo, la conducta social del paciente parece estar abstraída, confusa y sin sentido. Después, puede volver a funcionar al nivel anterior a la crisis o bien presentar síntomas de deterioro. Sobre esto van a influir factores psicosociales externos y variables internas que constituyen la vulnerabilidad. Según investigaciones recientes (Penn y Mueser, 1996), a los dos años del comienzo de la enfermedad un $40 \%$ de los pacientes ya muestran déficits funcionales graves $y$ otro $30 \%$ de mediana gravedad. Al no contar con habilidades sociales, estos enfermos no pueden vivir experiencias de interacción social y de vínculos interpersonales como refuerzos positivos. El escaso procesamiento de las situaciones sociales tiene significativa importancia en que el paciente presente deterioro en la capacidad de escuchar, entender, identificar sentimientos de los otros, concentrarse en un tema de discusión y evitar cambios abruptos, coordinar armónicamente conducta verbal y analógica, aceptar críticas, etc. Todo ello constituye en los pacientes esquizofrénicos lo que se conoce con el nombre de «síndrome de alteración de la capacidad de relación", con dificultades para entablar nuevas relaciones, problemas en el trabajo, etc.

Se ha documentado la eficacia del E.H.S. en más de 50 estudios de investigación desde finales de los años 70 en los que se ha puesto de manifiesto que el 
funcionamiento social es un importante predictor del pronóstico del paciente esquizofrénico (Liberman, 1982).

Siguiendo a Shriqui y Nasrallah (1996), el E.H.S, se puede agrupar en tres modelos: (1) El Modelo Básico, para enseñar a los pacientes habilidades sociales verbales y no verbales específicas (contacto visual, sonrisa, volumen de voz, formulación de preguntas, saludo, etc.). (2) El Modelo de Resolución de Problemas, el cual aporta estrategias para capacitar a los pacientes a manejar situaciones sociales y vitales no ensayadas que tienen que ver con el manejo de la medicación, el dinero, el cuidado y aseo personal y la resolución de problemas sociales. (3) El Modelo de Focalización de la Atención, desarrollado para pacientes con graves alteraciones cognitivas (memoria y atención) y que se caracteriza por la utilización de técnicas de adiestramiento breves (juego de roles, modelado, refuerzo positivo y negativo).

La eficacia del entrenamiento en estas habilidades ha sido refrendada en varios estudios (Bellack y Hersen, 1988; Wallace, 1980; Hersen, 1979) con relación a una mejora en la adaptación y competencia social de los pacientes, la existencia de periodos más largos sin recaídas y las autoevaluaciones de asertividad. No obstante, la evaluación de la generalización y mantenimiento de efectos de la terapia no ha sido tan satisfactoria ya que existen dudas respecto a la duración óptima del adiestramiento, la existencia de objetivos conductuales entrenables diferentes en las distintas investigaciones, la falta de control de variables ajenas al entrenamiento, etc. Se plantean interrogantes sobre si una mejoría durante el adiestramiento implica una mejora funcional en la comunidad y sobre la eficacia para reducir sintomatología psicótica. En ocasiones, quizá sean las diferencias en los planteamientos metodológicos de las distintas investigaciones (selección de muestras de pacientes, técnicas de evaluación,...), las que están dificultando la aparición de resultados que vayan en la misma línea (Aldaz y Vázquez, 1996). Sin embargo existen estudios que señalan una generalización al medio natural (Wong y Woolsey, 1989) de las condiciones sociales apropiadas adquiridas o incrementadas a través de los programas de E.H.S.

\section{INTERVENCIONES DIRIGIDAS AL CONTROL DE SÍNTOMAS POSITIVOS}

Tal y como hemos apuntado más arriba, los síntomas positivos reflejan la distorsión o exageración de las funciones psicológicas, mientras que los síntomas negativos se refieren a la pérdida o disminución de dichas funciones. Los principales síntomas positivos asociados a la esquizofrenia son las alucinaciones auditivas en segunda y tercera persona, los delirios (de control, de referencia y paranoides) y los fenómenos extraños al Yo (inserción del pensamiento, transmisión del pensamiento, eco del pensamiento y robo del pensamiento) (Frith, 1995).

Dado que, a pesar del tratamiento farmacológico, en algunos pacientes persisten los síntomas positivos tras una fase aguda o brote de la enfermedad, se vio necesario buscar otros procedimientos que permitieran al paciente controlar, aunque sea de forma parcial, algunos de sus síntomas positivos: las alucinaciones y los delirios.

\section{Intervenciones psicológicas sobre las alucinaciones}

En esta área se han venido realizando intervenciones que utilizan técnicas conductuales clásicas sustentadas por modelos teóricos potentes (procedimientos 
operantes, desensibilización sistemática, parada de pensamiento) hasta otras técnicas que, resultando eficaces en su aplicación, no queda totalmente claro el soporte teórico subyacente.

Slade (1988) realiza una exhaustiva revisión de los estudios experimentales y clínicos de tratamiento psicológico, principalmente de corte conductual, de las alucinaciones en pacientes esquizofrénicos. Para este autor, una de las formas de intervención sobre las alucinaciones ha sido realizada mediante procedimientos conductuales operantes. Las investigaciones realizadas en esta línea parten de hacer operativa la conducta alucinatoria de forma que se permita su observación y registro (por ejemplo, centrarse en las conductas verbales o motoras que se valoran asociadas a la experiencia alucinatoria, tales como soliloquios, musitaciones, gritos, rituales compulsivos, etc.), para pasar después a un análisis funcional de la conducta problema objeto de la modificación.

Las técnicas utilizadas han sido diversas: (1) Refuerzo positivo de conductas adaptativas incompatibles con aquellas asociadas a la experiencia alucinatoria (Nyddegger, 1972); (2) Tiempo fuera contingente a las conductas alucinatorias (Heynes y Geddy, 1973). (3) Combinaciones de técnicas tratando de realizar un control de contingencias.

Anderson y Allpert (1974) aplicaron el castigo positivo contingentemente a la conducta operativizada como alucinatoria y refuerzo social a aquellas conductas que resultaban adaptativas y eran incompatibles con la alucinatoria. Los resultados mostraron una reducción de la tasa de conducta alucinatoria sumándose a esto, en algunos casos, un incremento de conductas adaptativas y competitivas con las alucinatorias e incluso en algunos estudios se informa de la desaparición de la conducta alucinatoria y problemas asociados (Nydegger, 1972).
Las alucinaciones también se han tratado a través de la desensibilización sistemática. La lógica que subyace a la utilización de esta técnica en el tratamiento de las alucinaciones es que la aparición de "las voces» se asocia a situaciones estresantes (situaciones sociales o interpersonales y situaciones de convivencia familiar) que conllevan un aumento del arousal y tensión emocional. La hipótesis es que si se consigue una desensibilización a los estímulos estresantes condicionados, se producirá una reducción de la experiencia alucinatoria.

Los resultados de las investigaciones (Slade, 1972, 1973) han sido exitosos en cuanto a la reducción en frecuencia de las alucinaciones autoinformadas o desaparición de las mismas. Paralelamente se ha realizado algún trabajo en el cual la desensibilización sistemática se ha orientado a modificar la cualidad de la "voz" desde una hostil a una amistosa, obteniéndose resultados positivos. En esta línea, Siegel (1975) a través de un análisis conductual averigua que las alucinaciones auditivas imperativas en un paciente le producian unos altos índices de ansiedad. Su intervención se orientó a desensibilizar al paciente de las voces generadoras de ansiedad. El resultado obtenido fue que el tono hostil de las alucinaciones cambió por otro amistoso que no molestaba al paciente a pesar de persistir la tasa de dichas alucinaciones.

Diversos autores han utilizado la técnica de la parada de pensamiento, de forma flexible e imaginativa, para el control de la conducta alucinatoria. En líneas generales se trata de pasar de un control abierto y externo de las alucinaciones por parte del terapeuta a un control encubierto interno del propio paciente (Johnson, Gilmore y Shenay, 1983) o bien a un control abierto propio (Lamontgne, Audel y Elic, 1983). Allen, Halperin y Fried (1985) plantearon la hipótesis de que el procedimiento de la parada de 
pensamiento reducía la duración de las alucinaciones, pero no así la frecuencia, la cual se vería afectada por una táctica distractora. Esta hipótesis se puso a prueba en un estudio de caso único, corroborándose por los resultados obtenidos aunque con una falta de generalización de los efectos positivos.

Por otra parte, dada la relación entre la estimulación y las alucinaciones, se han utilizado una serie de procedimientos orientados a manipular las variables estimulares que rodean al paciente a la espera de que ello modifique algunos de los parámetros de la conducta alucinatoria. De acuerdo a estos procedimientos, investigadores y clínicos han tratado de incidir sobre la aparición de las alucinaciones a través de la reducción del input sensorial de los pacientes. Harris (1979) obtuvo resultados positivos en la reducción de las alucinaciones auditivas de pacientes esquizofrénicos sometiéndoles a breves periodos de deprivación sensorial. Sin embargo, la debilidad metodológica del estudio y la evidencia en sentido contrario de otros estudios impide obtener conclusiones definitivas.

Otro procedimiento dirigido a controlar las alucinaciones a través de la manipulación de variables estimulares es la contraestimulación. Esta técnica se basa en considerar a las alucinaciones como un tipo de estimulación interna y trataría de que otra estimulación más potente contrarreste o interfiera la anterior de forma que se reduzca. Margo, Hensley y Slade (1981) informan de una reducción de la tasa de experiencias alucinatorias durante los ensayos de estudio en pacientes esquizofrénicos a través de la presentación de estimulación visual y auditiva. Sin embargo, no se produjo una generalización del control de las alucinaciones a situaciones cotidianas distintas de las sesiones de estudio. Turner y cols (1976) en un estudio sobre la generalización de los efectos de reducción de las alucinaciones a través de la contraestimulación con una conversación social, una actividad motora y estimulación aversiva, hallaron que solo se producía dicha generalización en el caso de la estimulación eléctrica aversiva.

Otros estudios han utilizado la automonitorización del paciente sobre sus experiencias alucinatorias con objeto de controlarlas, encontrándose hallazgos distintos. La simple contabilización de las frecuencias diaria de la experiencia alucinatoria no se sigue de ningún efecto (Rybee y Kinch, 1973) o incluso puede producir un aumento de la frecuencia informada (Moser, 1974). Sin embargo, se ha comprobado que la Auto-observación y valoración concurrente de las alucinaciones realizada a través de un registro automático o escrito asociada a otras técnicas contingentes a las experiencias alucinatorias, como la sensibilización encubierta o la saciación, demostraron una disminución de las experiencias alucinatorias (Moser, 1974; Glaister, 1985).

La estimulación aversiva contingente a la experiencia alucinatoria informada por los pacientes ha demostrado tener una efectividad inmediata en la reducción de las alucinaciones (Watts y Clements, 1971; Weingaertner, 1971). No obstante, dicha reducción no se mantiene en la vida cotidiana, además de ser una técnica que conlleva efectos colaterales no deseables.

Por otra parte, la técnica propuesta por Greene (1978) sobre la transferencia e integración interhemisférica defectuosa en la esquizofrenia ha sugerido el uso de tapones en el oído para el control de las alucinaciones. En las investigaciones realizadas, se informa de una reducción significativa de las mismas (Dones, Frith y Owers, 1986; Birchwood, 1986), aunque no se pueden explicar claramente en términos de la teoría original de Greene.

Distintos autores indican que las alucinaciones pueden estar parcialmente influidas por las creencias y expectativas que el 
paciente tenga sobre las mismas (Minzt y Allpert, 1972; Young, Benthal, Slade y Dewey, 1987). En esta línea Greene (1978) señala la importancia en la experiencia alucinatoria de los pacientes la atribución de un locus de control externo de la misma; es decir, los pacientes que tienen alucinaciones las atribuyen a factores externos y no controlables por ellos. La propuesta de la Terapia de la Primera Persona del Singular (Greene, 1978) es la de "educar» al paciente para que atribuya la experiencia alucinatoria a un locus de control interno. Desde esta perspectiva se animaba a los pacientes para que adoptaran la primera persona del singular y se refirieran a sus voces como «hablando consigo mismo». La utilización de esta técnica ha tenido efectos beneficiosos en cuanto a la reducción de la frecuencia de las alucinaciones. Sin embargo, los resultados hallados han fluctuado bastante de una investigación a otra y los cambios obtenidos no pueden atribuirse a la utilización de esta técnica en exclusiva, dado que se han puesto en marcha varios procedimientos con objeto de controlar las alucinaciones al mismo tiempo (Fowler, 1986).

Slade y Bental (1988) señalan que los éxitos hallados en la reducción de las alucinaciones en los pacientes esquizofrénicos a través de los tratamientos psicológicos se pueden explicar por tres procesos: la focalización de la atención, la reducción de la ansiedad y la distracción o contraestimulación; coincidiendo dichos factores con los mecanismos de afrontamiento espontaneo que utilizan los pacientes para el control de sus experiencias alucinatorias (Fallon y Talbot, 1981; Tarrier, 1987).

Intervenciones psicológicas sobre la conducta delirante

Los estudios que han utilizado las técnicas operantes para la modificación de la conducta delirante parten de que la misma está mantenida por las consecuencias que le suceden y que aquella puede disminuirse o eliminarse a través de la modificación de las contingencias. Ayllon y Haugton (1964) publicaron la primera demostración experimental del control operante del habla delirante. Sobre una línea base tomada durante tres semanas se muestra cómo las contingencias discriminativas (refuerzos materiales y sociales) al habla delirante y neutra, conlleva un incremento en todos los casos. Es decir, este estudio constituye una prueba de generalización del condicionamiento verbal al habla delirante.

Los primeros estudios que utilizaban técnicas operantes para la modificación de la conducta delirante (Ayllon y Michael, 1959; Lindsley, 1960; Kennedy, 1964; Rashkis, 1966) adolecen de poco rigor metodológico. Las técnicas utilizadas por estos autores eran el refuerzo positivo (material y/o social), el castigo positivo (desaprobación social) y la extinción (ausencia de consecuencias) contingente a la conducta delirante. Los resultados hallados van en la dirección de un aumento de la conducta verbal normalizada y una disminución de la conducta delirante durante la fase experimental sin que se aporten datos sobre la generalización de tales efectos.

Winvcze, Leitemberg y Agras (1972) trataron de modificar la conducta delirante de diez pacientes diagnosticados de esquizofrenia paranoide a través de la utilización del «feedback» y reforzamiento con fichas ante respuesta correctas (no delirantes). Estos autores encontraron una disminución de la conducta delirante en las fases en las que se utilizaba el refuerzo contingentemente, sin que se hallara efecto cuando se utilizaba sólo la instrucción sin refuerzo. También se comprobó una ausencia de generalización de tales efectos a otras circunstancias. 
En general, los resultados del control operante de la conducta delirante van en la dirección de un aumento de la conducta verbal normalizada y una disminución de la conducta delirante durante la fase experimental. Las limitaciones encontradas en estos estudios giran en torno a las dificultades de generalización de los resultados a otros ambientes y situaciones no experimentales y al hecho de que muchos son de caso único. Sin embargo, existen estudios que ponen de manifiesto, a parte del control operante de la conducta delirante, la generalización espaciotemporal de los efectos del tratamiento (Liberman y cols.) , 1975 buscar).

Por otra parte, la Desensibilización Sistemática y la relajación también ha sido utilizada para el control del habla delirante del paciente esquizofrénico con sintomatología psicótica positiva (Cowden y Ford, 1962; Zeisset, 1968). Aunque existen importantes diferencias metodológicas entre los estudios realizados en esta línea, generalmente se trata de desensibilizar al paciente en aquellas situaciones que le son ansiógenas y que habitualmente se relacionan con su temática delirante. Para ello se procede de la forma habitual de la aplicación de esta técnica. Los resultados obtenidos difieren bastante entre sí aunque aparece una tendencia a disminuir el habla delirante y otros síntomas psicóticos en los pacientes tratados con esta técnica. Sin embargo, estos estudios adolecen en su mayoría de un control experimental y de falta de información sobre la generalización de los efectos del tratamiento.

También se han realizado intervenciones cognitivas orientadas al tratamiento de los delirios. Autores actuales han argumentado que los delirios de pacientes psiquiátricos deberían considerarse un continuo que iría desde las creencias de la vida diaria hasta las extrañas creencias de los pacientes más perturbados (Harrow, Rattenbury y Stoll, 1988).
Los abordajes para la modificación de las creencias delirantes han sido diversos. Se ha utilizado la Terapia Atribucional que supone la «modificación de creencias» de una forma graduada empezando por las más periféricas para después, poco a poco, ir trabajando creencias centrales (Watts, Powell y Austin, 1973). También se han puesto en práctica Pruebas de Realidad, en las que se animaba a los pacientes a construir pruebas críticas para evaluar sus creencias (Chadwick y Lowe, 1990) y Pruebas de Monitorización de la Realidad, consistente en la Auto-observación y valoración a través de un registrador automático. Asimismo, se ha utilizado estrategias de afrontamiento, que se refieren a la utilización espontánea de recursos cognitivos y conductuales para contrarrestar la angustia causada por los síntomas psicóticos.

Los resultados encontrados han sido positivos en la reducción de la convicción del pensamiento delirante y apoyan la hipótesis defendida por varios autores de que las anormalidades atribucionales y de autoesquema juegan un papel central en los delirios persecutorios (Bentall, 1995). Al mismo tiempo, se ha puesto de manifiesto que pacientes con este tipo de delirios prestan mayor atención a la información relacionada con temas de amenaza y la recuerdan con preferencia (Bentall y Kaney, 1989). Otros autores, como John y Dogson (1994) proponen que los pacientes con delirios podrían tener dificultades a la hora de realizar un análisis adecuado de la información de cara a la solución de problemas.

\section{INTERVENCIONES DIRIGIDAS A LA PREVENCIÓN DE RECAÍDAS}

La esquizofrenia es un trastorno crónico que cursa como un proceso con crisis de descompensaciones psicopatoloógicas 
tras la cual el paciente puede volver al nivel de funcionamiento premórbido o experimentar un deterioro respecto al funcionamiento previo a la crisis, llegando algunos casos a un defecto progresivo.

La prevención o alivio de la recaída es importante para el futuro bienestar del paciente con esquizofrenia. Se sabe que cada recaída aumenta la probabilidad de recidivas futuras, síntomas residuales y consecuente discapacidad social; de ahí que se hayan puesto en marcha distintos tipos de programas, tales como Programas de Intervención Precoz, Programas de Intervención con Familias e intervenciones dirigidas a potenciar los recursos personales de los pacientes.

\section{Programas de Intervención Precoz}

La ižiervención precoz es un abordaje dirigido principalmente a personas jovenes y vulnerables, y tiene por objeto disminuir o controlar, en la medida de lo posible, el deterioro psicológico y sociofamiliar que se pueda producir en el paciente esquizofrénico.

Parece demostrado que la forma en que transcurren los primeros años de enfermedad es un momento clave para el desarrollo posterior de esta. Por tanto, la intervención en estas primeras etapas está claramente indicada. Dicha intervención tiene su aplicación en dos momentos: antes o durante el primer episodio de esquizofrenia y en la detección precoz de signos que apuntan a una recaída en individuos con vulnerabilidad a la esquizofrenia.

Estudios realizados en pacientes con un primer episodio de esquizofrenia sugieren que la intervención antes o durante éste, tiene un efecto muy importante sobre la vulnerabilidad futura a las recaídas, en comparación con intervenciones posteriores (Harding y Zahniser, 1994).
Muchos pacientes y familiares pueden reconocer los signos prodrómicos característicos de descompensación que ocurren entre la segunda y la cuarta semana anterior a la recaída. De ahí que algunos autores (Birchwood, Smith, Mc Millan, Hogg, Prasad, Harvey y Bering, 1989) hayan puesto en marcha programas de entrenamiento en la detección de tales signos dirigidos a pacientes y familiares, con el objetivo de favorecer intervenciones tempranas que eviten, en la medida de lo posible, la recaída del paciente y el consiguiente internamiento en una unidad de hospitalización.

Birchwood et al. (1989), señalan que los últimos estudios realizados al respecto parecen indicar que más que la detección de signos prodrómicos universales, es más apropiado el estudio personalizado del pródromo en cada paciente. A éste se le ha denominado firma de recaída y estaría constituida por los síntomas nucleares junto a las características específicas del sujeto.

\section{Programas de Intervención con Familias}

$\mathrm{El}$ ambiente familiar ha sido una constante a la hora de abordar el estudio de la esquizofrenia. En este sentido, los estudios realizados han pasado de considerar el papel de la familia como un factor etiológico a considerarlo como un factor potencialmente protector que puede influir en el curso del trastorno.

La hipótesis de trabajo, desde esta perspectiva, alude a que si pueden identificarse en el ambiente familiar factores que influyan en las recidivas su modificación contribuiría a disminuir las mismas. Entre los factores identificados destacan los altos índices de emoción expresada en los ambientes familiares y el grado de conocimiento que tenga la familia sobre la enfermedad y su manejo. 
Hacia ambos factores se ha orientado la elaboración las intervenciones.

Intervenciones familiares orientadas a reducir los altos índices de emoción expresada.

El término Emoción Expresada (EE) tiene su origen en los trabajos de Brown (1958, 1966), quien define el constructo a partir de tres factores: comentarios críticos, sobre-involucración y hostilidad manifestada hacia el paciente.

La hipótesis de partida de cara a la intervención con familias de pacientes esquizofrénicos con estas características señala que si las actitudes familiares con elevada EE pueden ser determinantes en las recaídas, entonces el cambio de tales actitudes a través del trabajo con familias podría hacer decrecer tal rango de recaídas.

Son muchos los estudios que han encontrado la relación significativa entre el retorno del paciente a la convivencia familiar con alto rango de EE y sus recaídas (Tarrier y Barrowclough, 1988). Sin embargo, se tienen pocos datos acerca de cómo la elevada EE en el ambiente familiar podría estar influyendo en las mismas. Esto plantea ciertos problemas desde el punto de vista de la intervención, ya que si no se puede identificar la forma precisa en que se dan este tipo de interacciones estresantes dentro de la familia, es difícil seleccionar los comportamientos que deberían modificarse. Siguiendo a estos mismos autores, son varias las suposiciones planteadas acerca de la clase de problemas que puedan estar interviniendo en estas familias. Entre éstos, se incluyen: los conocimientos erróneos que la familia pueda manejar sobre la enfermedad, los cuales podrían desembocar en conflictos o expectativas no realistas sobre el paciente y las dificultades en cuanto a la resolución de problemas, comunicación o afrontamiento de situaciones difíciles dentro de la convivencia familiar.

Tarrier (1991), tras revisar los estudios realizados sobre intervenciones familiares de pacientes esquizofrénicos dirigidas a reducir los altos índices de EE, ponen de manifiesto: (1) Una reducción de los porcentajes de recaída en comparación con los grupos control (Fallon, Boyd, McGill, Ranzani, Moss y Guildermannl, 1982; Leff, Kuipers, Berkowitz, EberleinFries y Sturgeon, 1982; Leff, Berkowtz, Shavit, Stracham, Glass y Vaughn, 1990; Hogarty, Anderson, Reiss, Kornblith, Greenwald, Javaan y Madonia, 1986; Tarrier, Barrowclough, Vaughn, Bamrah, Porceddu, Watts y Freeman, 1988). (2) Los beneficios obtenidos tras la intervención se mantienen en dos años de seguimiento, con un incremento de las recaídas si la intervención no es continuada (Fallon, 1985). (3) El rango menor de recaídas se asociaba a un mayor descenso en el nivel de EE (Hogarty, McEvoy, Munezt, DiBarry, Bartone, Cather, Cooley, Ulhrich, Carter y Madonia, 1988; Tarrier et al. , 1988; Leff et al. , 1989). (4) Las intervenciones que incorporan programas educacionales y medidas profilácticas favorecen la reducción del estrés o tensión dentro del ámbito familiar (Tarrier, 1991).

Aunque las recaídas han sido la variable pronóstico clave en este tipo de estudios, parece ser que las medidas de resultados múltiples, como funcionamiento social, carga familiar, satisfacción del consumidor y otras, son más apropiada para evaluar la eficacia del tratamiento con familias (Barrowclough y Tarrier, 1984).

\section{Programas Psicoeducativos}

Los programas psicoeducativos para pacientes esquizofrénicos tienen como principal objetivo el abordaje y apoyo del paciente y los miembros de su familia 
para reducir el efecto que los estímulos estresores tienen sobre ambos. El propósito último es el de facilitar la reinserción social del paciente tras el alta hospitalaria, disminuir la probabilidad de recaída y/o mejorar la calidad de vida del paciente y su familia.

Hasta ahora, muchos de estos programas se habían centrado exclusivamente en transmitir información a la familia y paciente sobre la enfermedad. Parece demostrado que este tipo de intervenciones simplemente educativas no resultan eficaces para la familia más allá de la ampliación de conocimientos sobre esquizofrenia y satisfacción por el trato recibido. En ausencia de un entrenamiento más específico en reducción de estrés y solución de problemas e intervenciones más profundas en la dinámica familiar, estas acciones no proveen a las familias de habilidades de afrontamiento y soporte suficientes como para modificar la evolución natural de la esquizofrenia (Aldaz y Vázquez, 1996).

A los estudios anteriormente citados se unen otros con mayor rigor metodológico que introducen aproximaciones de afrontamiento al estrés. Hay varios autores que se han centrado en la aplicación de programas psicoeducativos en sentido amplio para pacientes esquizofrénicos, derivándose de tales experiencias la evidencia de la efectividad de los mismos (Anderson, Reiss y Hogarty, 1986; Falloon, Boyd y McGuill, 1984; Leff, Kuipers, Berkowitz y Sturgeon, 1985; Tarrier et al., 1988).

Sanz y Cañive (1996) hacen una revisión de algunos de los principios generales comunes a este tipo de intervenciones y destacan los siguientes: (1) Los trastornos esquizofrénicos son entendidos como una patología con evolución generalmente crónica, que afectará al anormal desarrollo vital del enfermo. (2) Las intervenciones familiares se consideran tan solo una parte de las acciones que deben realizarse en este tipo de cuadros. (3) No se aplica un esquema rígido al aproximarse a la familia. Es necesario tener en cuenta numerosos factores, desde el grado de disfunción familiar desarrollada al tipo de habilidades de afrontamiento disponibles. (4) No se implica a la familia en la etiología de la esquizofrenia. (5) El desarrollo de la alianza terapéutica resulta esencial. Se fomenta una clara alianza con los familiares por parte del equipo terapéutico, promoviendo la visión de trabajo común.

Entre los programas mejor estructurados y que han utilizado una metodología más rigurosa se encuentran los siguientes: Programa de Intervención de Goldstein (1976), la "Terapia Conductual Familiar» de Fallon, Boyd y McGuill (1984), el Modelo Psicoeducacional de Anderson et al. (1986), el paquete de intervenciones sociales del grupo de Leff (1985), intervenciones de Tarrier et al. (1988).

Asimismo, en la revisión anteriormente mencionada, los autores encuentran varios elementos comunes a dichos programas: una evaluación sistemática de las características familiares; una actitud de aproximación positiva, empática, buscando una alianza de trabajo con la familia y el paciente; un espacio en el tratamiento dedicado a la información sobre la enfermedad; una estructura estable de tratamiento a la familia; un modo de fomentar relaciones con otros familiares en situación similar; y, el entrenamiento en la mejora de la comunicación y en el manejo de los problemas.

Intervenciones dirigidas a potenciar los recursos personales de los pacientes

Entre este tipo de intervenciones se pueden incluir las que han entrenado a los pacientes esquizofrénicos en habilidades de solución de problemas de la vida diaria y habilidades sociales, ya comentado anteriormente. 
Harsen et al. (1985) realizaron un programa de entrenamiento de solución de problemas de la vida diaria con siete pacientes de un plan de hospitalización parcial. Los resultados mostraron una mejoría en la solución de problemas en situaciones entrenadas y la generalización de las habilidades adquiridas a situaciones novedosas, quedando demostrada la validación social de las mejorías experimentadas a través de la comparación entre el grupo de pacientes entrenados y un grupo control de sujetos sin trastorno psiquiátrico.

Por otra parte, se sabe que las situaciones y circunstancias estresantes actúan como factores facilitadores de las descompensaciones psicopatológicas en los pacientes esquizofrénicos, que en ocasiones desencadenan hospitalizaciones. Es por ello importante actuar a este nivel en el intento de entrenar al paciente a reducir los efectos del estrés y a crear ambientes menos estresantes. En este sentido se han utilizado una serie de técnicas, que trabajadas conjuntamente favorecen un mejor manejo del estrés. Zeisset (1968) ya entrenó a los pacientes en relajación con el objetivo de reducir el arousal a través de la inducción sistemática de la relajación muscular. Otros autores (Slade, 1977; Hogan, 1966; Almbaugh, 1971) utilizaron la desensibilización sistemática orientada a reducir el miedo y ansiedad desencadenados por estímulos condicionados aversivamente. Obtuvieron generalización de sus efectos conseguidos, así como resultados positivos utilizando variantes de esta técnica.

\section{LA REHABILITACIÓN COGNITIVA EN LA ESQUIZOFRENIA}

Desde la Psicología Cognitiva y Experimental se están desarrollando investigaciones sobre las disfunciones cognitivas de los pacientes con esquizofrenia con el fin de elaborar programas destinados a su tratamiento. Los trastornos cognitivos se consideran una característica fundamental de la esquizofrenia (Brenner, 1983; George y Neufeld, 1985 y Fallon, 1986). En estos pacientes se aprecian importantes alteraciones cognitivas en áreas tan distintas como la atención (Ruiz Vargas, 1987), el funcionamiento sensoperceptivo (Bentall, 1990), la memoria (Neufeld, 1991) o en el pensamiento y lenguaje (Cohen, Targ, Servan-Schreiber y Spiegel, 1992; Staffy, 1993).

La investigación experimental y neuropsicológica de las dos últimas décadas sobre el déficit psicológico central en la esquizofrenia está bien documentada (revisiones de Lang y Buss, 1965; Nuechterlein y Dawson, 1984). Tal déficit parece independiente de circunstancias ajenas al trastorno (por ejem. la medicación o la institucionalización) y se presenta en todos los pacientes esquizofrénicos. Sin embargo, su intensidad varía de unos a otros, siendo el déficit cognitivo más marcado en los pacientes con predominio de sintomatología negativa, permitiendo incluso establecer comparaciones con algunos síndromes neurológicos (Lidde, 1990). Las funciones que se ven afectadas principalmente son la atención y memoria, en particular la verbal, la memoria de trabajo y la memoria a corto plazo (Jarne, Costa y Sanz, 1995).

Ha sido el paradigma Cognitivo del Procesamiento de la Información desde donde se han realizado las mayores aportaciones al estudio de las funciones cognitivas de los esquizofrénicos. La investigación desde este paradigma ha avanzado en la última década hasta relacionar las disfunciones cognitivas con la clínica esquizofrénica, entendiéndolas como variables personales determinantes en la vulnerabilidad para presentar un episodio esquizofrénico (Frith, 1992).

Las investigaciones sobre el procesamiento de información en pacientes 
esquizofrénicos muestran que existen disfunciones en distintas áreas, tales como en los problemas en la selección de estímulos relevantes y en el filtrado de estímulos irrelevantes, dificultades para mantener y dirigir la atención focalizada, para disponer de la información previamente almacenada y para identificar estímulos, disminución de la capacidad de abstracción, de derivar conclusiones deductivas y análogas correctas o de evocar la respuesta apropiada debido a la interferencia con otras respuestas competidoras (Ruiz Vargas, 1987; Aldaz y Vázquez, 1996). Estos pacientes muestran un rendimiento deficitario en tareas bajo control consciente que demandan gran cantidad de capacidad de procesamiento y de atención, mientras que en aquellas que no necesitan de un control consciente y pueden llevarse a cabo con un nivel mínimo de atención muestran un rendimiento óptimo.

Aunque desde la década de los 60 se han hecho aproximaciones a la rehabilitación cognitiva de pacientes esquizofrénicos (Wagner, 1968; Adams, Brantley, Malatesta y Turkat, 1981), no fue hasta la década de los 80 y 90 cuando aparece con mayor importancia esta área de estudio e intervención. Esto respondió a la idea de que la rehabilitación cognitiva, como primer paso en el proceso terapéutico, mejoraría el funcionamiento psicosocial de un paciente esquizofrénico (Braff, 1985; Brenner, Holder, Genner, Roder y Corrigan, 1992).

Vázquez, Florit y López (1996) abordan la intervención en rehabilitación cognitiva en pacientes esquizofrénicos. Señalan las características generales que deben cumplir los programas de rehabilitación cognitiva y describen algunos de los programas y técnicas más usadas para el entrenamiento de las habilidades cognitivas, centrándose en la atención y memoria.

La rehabilitación cognitiva parte de que son los déficits cognitivos de los pacientes esquizofrénicos la base de los trastornos de conducta que se observan a un nivel psicosocial, y es por lo que proponen una intervención en un nivel inferior más básico (cognitivo) a la espera de que ello produzca modificaciones en niveles superiores más complejos (por ejemplo, psicosociales).

Los estudios específicos sobre la rehabilitación cognitiva en la esquizofrenia han sido escasos. Entre éstos destaca el de Adams et al. (1981) que diseñaron un programa terapéutico dirigido a aumentar la atención y disminuir la distraibilidad y otros déficits cognitivos. La hipótesis realizada por estos autores es que «el paciente no es capaz de discriminar adecuadamente entre estímulos internos y externos y tiene dificultad para atender a las claves verbales y no verbales, resultando todo ello en un déficits en habilidades sociales». El programa diseñado por estos autores fue de ámbito hospitalario con dos grandes áreas de intervención. Por un lado, entrenamiento en atención a estímulos externos apropiados con diversas estimulaciones distractoras (auditivas, visuales, conversacionales e interpersonales ) y, por otra parte, entrenamiento en la atención a estímulos internos apropiados. Las técnicas conductuales utilizadas fueron variadas, tales como modelado, "role playing", "feedback», parada de pensamiento e instrucciones. Los hallazgos obtenidos tras finalizar el programa de intervención y a los seis meses de seguimiento constataban una ausencia de desórdenes de pensamiento, una mejoría en la efectividad social y una ausencia de sintomatología depresiva. Estos resultados vendrían a confirmar la hipótesis de la mediación del déficit en el procesamiento de información, fundamentalmente atencional, en la clínica que manifiesta el paciente esquizofrénico; si bien no existe constancia de que tal relación sea causal ni lineal, haciéndose necesaria más investigación en esta área. 
Benedict et al. (1989) realizaron un programa de intervención dirigido a mejorar la atención de un grupo de pacientes esquizofrénicos crónicos, diferenciando un grupo experimental de otro control. El programa perseguía mejorar el tiempo de reacción mediante tareas computacionales que incluían entrenamiento en velocidad de procesamiento de la información y vigilancia y tareas mnésicas, de formación de conceptos y solución de problemas. Cada tarea estaba graduada de menor a mayor dificultad. Los resultados obtenidos mostraron una mejoría de los tiempos de reacción en el grupo experimental, sin que se ofrecieran datos sobre la generalización a la vida cotidiana.

Otra experiencia sobre la rehabilitación cognitiva en pacientes esquizofrénicos es la llevada a cabo por Brenner et al. (1992) en su Terapia Psicológica Integrada (Integrated Psychological Therapy, IPT). El modelo teórico en el que se basa la IPT parte de considerar que la conducta está jerárquicamente organizada, de forma que los déficits cognitivos más básicos (atención y memoria) influyen en una disfunción más compleja (formación de conceptos y recuperación); determinando ello una disminución de las habilidades de afrontamiento $y$, consecuentemente, un peor manejo de las situaciones de estrés. Todo ello se traduce en una disminución de las capacidades del sujeto y en la creación de una relación circular entre las variables anteriormente señaladas e implicadas en el comportamiento del paciente.

El programa terapéutico que proponen estos autores parte de desarrollar los déficits cognitivos elementales (concentración, percepción y memoria) y las disfunciones cognitivas complejas que estos determinan (formación de conceptos, capacidad de abstracción) y , posteriormente, practicar formas más complejas de habilidades y conducta social. El programa consta de una secuencia de cinco subprogramas: diferenciación cognitiva, percepción social, comunicación verbal, habilidades sociales y resolución de problemas interpersonales. La IPT es de aplicación grupal (de 5 a 7 pacientes) en sesiones de 30 a 60 minutos, tres veces por semana y en un periodo de unos 3 meses. Durante el curso del programa va aumentando el grado de exigencia hacia el paciente y hacia el grupo tanto en lo que se refiere al contenido como a toda la estructura de la terapia. $\mathrm{Su}$ método, al inicio del programa, es muy directivo, y estructurado pasando a una menor dirección al final alentando la interacción espontánea, la iniciativa y la responsabilidad del paciente. Igualmente, al principio se empleará un material terapéutico neutro, que no representa estrés emocional alguno para los pacientes, para ir introduciendo progresivamente contenidos emocionalmente estresantes, enfatizando más el afrontamiento del estrés emocional.

Los resultados aportados por los autores de la IPT son favorables; la eficacia del programa pudo ser confirmada repetidas veces; sin embargo, otros autores dudan sobre si la mejoría experimentada por los pacientes es específica del tratamiento (Liberman, 1992).

Otras intervenciones también inciden en la rehabilitación de las funciones cognitivas de manera indirecta (Liberman, 1988). Se trata de desarrollar habilidades y capacidades, a través por ejemplo del entrenamiento en habilidades sociales o programas psicoeducativos, que impliquen una participación, y con ello desarrollo, de las funciones cognitivas.

\section{CONCLUSIONES}

La complejidad etiopatogénica, clínica y de tratamiento de la esquizofrenia hace necesario que la aproximación a la misma se realice desde un modelo integrador 
y de naturaleza biopsicosocial, partiendo de la integración de factores orgánicos, ambientales y psicosociales en las manifestaciones clínicas del trastorno. Es por ello que el abordaje terapéutico, en consonancia con el modelo conceptual multifactorial propuesto, debe orientarse a abordar todas y cada una de las áreas presumiblemente implicadas en el trastorno resultando inadecuado un abordaje parcial del mismo.

Los avances conseguidos con los fármacos antipsicóticos en la remisión y prevención de crisis esquizofrénicas se deben complementar con una intervención psicológica dirigida al tratamiento y rehabilitación de estos pacientes.

Las aportaciones que la psicología ha ido haciendo, desde los años 60, al tratamiento de estos pacientes van muy unidas a los cambios en la política asistencial de los enfermos mentales que se produjeron durante esos años. Las nuevas directrices de esa política asistencial se vieron influidas por los principios de la psiquiatría comunitaria y las reformas basadas en la desinstitucionalización, lo que conllevó la apertura de la asistencia psiquiátrica a otras disciplinas cercanas, entre ellas la psicología. Sus aportaciones han ido encaminadas, fundamentalmente, a proporcionar habilidades y estrategias que permitan a los pacientes (y por extensión a sus familias) una mejora en su calidad de vida y una mayor adaptación a su entorno social evitando el aislamiento de estos enfermos. Los resultados de tratamientos psicosociales han demostrado su eficacia en:

- Mejorar la sintomatología presentada

- Aumentar la adherencia al tratamiento psicofarmacológico

- Rehabilitar habilidades perdidas por los efectos directos del trastorno o la institucionalización

- Dotar de recursos personales de afrontamiento a situaciones estresantes potencialmente desencadenantes de crisis esquizofrénicas

- Favorecer un mejor ajuste familiar y reducir las situaciones de interacción interpersonal elicitadoras de recaídas

Sin embargo, a pesar de la eficacia de los abordajes multidisciplinares constatada por los diferentes estudios, la realidad asistencial existente nos muestra que en la mayoría de las ocasiones, el tratamiento tiene un sesgo a favor de la farmacoterapia centrado, principalmente, en las crisis esquizofrénicas y con un carácter escasamente preventivo.

Son realmente pocos los casos en los que se realiza un diseño de intervención multiprofesional-multidisciplinar dirigido a actuar sobre los factores implicados en la aparición y mantenimiento de la clínica del paciente desde la primera crisis o detección de síntomas de inicio insidioso. Si bien es cierto que han sido varios los programas de tratamiento integral propuestos para la mejora de estos pacientes, también lo es que, en su mayoría, no están articulados de forma coordinada entre los distintos dispositivos de salud mental que atienden a estos enfermos como sería de desear, desaprovechándose de esta manera recursos terapéuticos disponibles. La presión asistencial a la que nos vemos sometidos los profesionales hace que, en ocasiones, los tratamientos persigan la rapidez a corto plazo en la consecución de sus objetivos y se olviden otros factores. En esa línea, pareciera que la "atención comunitaria» de estos pacientes está dejando de ser tal y parece estar volviendo a confinarse en los dispositivos creados para estos pacientes como «lugares de transición" en los cuales su estancia abarca cada vez periodos más largos de tiempo.

Nuestra propuesta a favor de un tratamiento global del paciente con esquizofrenia implica: 
- La intervención temprana sobre poblaciones de alto riesgo con indicadores positivos claros de vulnerabilidad

- La evaluación integral de estos pacientes

- El diseño de un abordaje multidisciplinar (médico, psicológico y social) individualizado según las necesidades detectadas en la evaluación del paciente.

- La designación de un terapeuta referente para el paciente y su familia, que coordine todas las intervenciones terapéuticas

- Respecto al funcionamiento de los dispositivos de atención a la salud mental, consideramos importante la existencia de programas terapéuticos diversos (psicoeducativos, de adquisición de habilidades sociales, de resolución de problemas, de control de síntomas, atención a la familia,...) articulados de forma coherente en el área sanitaria con el fin de realizar un adecuado aprovechamiento de los recursos terapéuticos existentes. Así mismo, consideramos imprescindible la coordinación estrecha entre los profesionales implicados en la valoración y tratamiento de estos pacientes y sus familias.

Mediante este trabajo hemos pretendido poner de manifiesto algunas de las aportaciones que, desde distintos ámbitos de la psicología, se vienen realizando en el abordaje terapéutico de pacientes con esquizofrenia. Consideramos muy importante se continúen elaborando programas integrales de tratamiento, así como que se siga el estudio de la efectividad de los mismos, con el fin de contribuir a la mejora de la calidad de vida de estos pacientes y sus familias.

\section{REFERENCIAS BIBLIOGRAFICAS}

Adams, H.E., Brantley, P.J., Malatesta, V., y Turkat, I.D. (1981). Modification of cogni- tive processes: a case estudy of schizophrenia. Journal of Consulting and Clinical Psychology, 49, 460-464.

Aldaz, J.A., y Vázquez, C. (Comps.) (1996). Esquizofrenia: fundamentos psicológicos y psiquiátricos de la rehabilitación. Madrid: Siglo XXI.

Anderson, C.M., Reiss, D.J., y Hogarty, G.E. (1986). Schizophrenia and the family. Nueva York: Guilford.

Atthowe, J.M., y Krasner, L. (1968). Preliminary report on the aplicattion of contingency reinforcement procedures (token economy) on a "chronic» psyquiatric ward. Journal of Abnormal Psychology, 73, 37-43.

Ayllon, T., y Azrin, N.T. (1968).The token economy: a motivational system for therapy and rehabilitation. Nueva York: Appelton-Century-Crofts.

Barrowclough, C., y Tarrier, N. (1984). Psychosocial interventions with families and their effects on the course of schizophrenia: a review. Psychological Medicine, 14, 629-642.

Bellack, A.S., y Hersen, M. (1988). Behavioral assessment: a practical handbook. Nueva York: Pergamon.

Bentall, R.P., y Kaney, S. (1989).Content-specific information processing and persecutory delusions: An investigation using the emotional stroop test. British Journal of Medical Psychology, 62, 355-364.

Bentall, R.P. (1990b). The illusion of reality: An review and integration of psychological research on hallucinations. Psychological Bulletin, 107, 82-95.

Bentall, R.P. (1996). La investigación psicológica sobre las alucinaciones y los delirios: psicopatología y aplicaciones para las estrategias del tratamiento. En J.A. Aldaz y C.Vázquez (Comps.), Esquizofrenia: fundamentos psicológicos y psiqyuiátricos de la rehabilitación. Madrid: Siglo XXI.

Birchwood, M.J.; Smith, J., Mc. Millan, F., Hogg, B., Prasad, R., Harvey, C., y Bering, $S$. (1989). Predicting relapse in schizophrenia: the development and implementation of an early signs monitoring system using patients and families as observers, a preliminary investigation. Psychological Medicine, 19, 649-656. 
Braff, D.L. (1985).Attention, habituation and information processing in psychiatric disorders. En R. Michels, J.O. Cavenar, H.K. Brodie et al. (Comps.), Psychiatry, 3, 1-10.

Brenner, H.D. (1983). Die Bedeutung experimental psychologischer forschung für Theorie und Therapie der Schizophrenie. En H.D. Brenner, E.R. Rey y W.G. Stramke (Eds.), Empirische Schizophrenieforschung. Bern, Huber.

Brenner, H.D., Hodel, B., Genner, R., Roder, V., y Corrigan, P.W. (1992). Biological and cognitive vulnerability factors in schizophrenia: Implications for treatment. British Journal of Psychiatry, 161, 154-163.

Brown, G.W., Carstairs, G.M., y Topping, G. (1958). Post-hospital adjustment of chronic mental patients. Lancet, 2 (7048), 685-689.

Brown, G.W., y Rutter, J. (1966). The measurement of families activities and relationships: A methodological study. Human Relations, 12, 241-263.

Chadwick, P., y Lowe, C.F. (1990). Measurement and modification of delusional beliefs.Journal of Consulting and Clinical Psychology, 58, 225-232.

Chapman, L.J., y Chapman, J.P. (1973). Disordered thought in schizophrenia. Nueva York: Appleton-Century-Crofts.

Ciompi, L. (1983). Schizophrenia deterioration discussion. British Journal of Psychiatry, 143, 77-84.

Cohen, J., Targ, E., Servan-Schreiber, D., y Spiegel, D. (1992). The fabric of thought disorder: a cognitive neuroscience approach to disturbances in the processing of context in schizophrenia. En D.J. Stein y J. Young (Comps.), Cognitive science and Clinical Disorders, 99-127, Nueva York: Academic.

Cowden, R.C., y Ford, L.I. (1962). Systematic desensitization with phobia schizophrenics. American Journal of Psychiatry, 119, 241-245.

D'Zurilla, T.J., y Golfried, M.R. (1971). Problem solving and behaviour modification. Journal of Abnormal Psychology, 78, 107126.

Falloon, I.R.H.; Boyd, J.L. y Mc Gill, C. (1984). Family care of schizophrenia. Nueva York: Guilford Press.
Falloon, I.R., Boyd, J.L., y Mc Gill, C. (1985). Family management in the prevention of morbility of schizophrenia. Archives of General Psychiatry, 42, 887-896.

Falloon, I.R., Boyd, J.L., Mc Gill, C., Ranzani, J., Moss, H.B., y Guildermann, A.M. (1982). Family management in the prevention of exhacerbation of schizophrenia. A contolled study. New England Journal of Medicine, 306, 1437-1440.

Frith, C.D. (1992). The cognitive neuropsychology of schizophrenia. London: Laurence Erlbaum Associates, Publishers.

Frith, C.D. (1995). La Esquizofrenia: un enfoque neuropsicológico cognitivo. Madrid: Ariel Psicología.

George, L. y Neufel, R.W. (1985). Cognition and symptomatology in schizophrenia. Schizophrenia Bulletin, 11 (2), 264-285.

Glaister, B. (1985). A case study of auditory hallucinations treated by satiation. Behaviour Research and Therapy, 23, 213-215.

Goldstein, G. (1986). The neuropsychology os schizophrenia. En I. Grant y K.M. Adams (Comps.), Neuropsychology asessment of neuropsychiatry disorders. Nueva York: Oxford University Press.

Green, M.F. (1992). Information processing in schizophrenia. En D.J. Kavanagh (Comp.), Schizophrenia: An overview and practical handbook. Nueva York: Chapman and Hall.

Greene, R.J. (1978). Auditory hallucination reduction: First person singular. Journal of Contemporary Psychotherapy, 9, 167-170.

Harding, C.M. y Zahniser, J.H. (1994). Empirical correction of seven myths about schizophrenia with implications for treatment. Acta Psychiatrica Scandinavica, 90, 140146.

Harris, A. (1979). Sensory deprivation and schizophrenia. Journal of Mental Science, 105, 235-237.

Harrow, M., Rattenbury, F., y Stoll, F. (1988). Schizophrenic delusions: an analysis of their persistence, of related premorbid ideas in three major dimensions. En T.F. Oltmans y B.A. Maher (Gomps.), Delusional beliefs. Nueva York: John Wiley.

Heaton, R.K., Bade, L.E., y Johnson, K.L. (1978). Neuropsychological test results associated with psychiatry disorders in adults. Psychological Bulletin, 85, 141-162. 
Hogarty, G., Anderson, C.M., Reiss, D.J., Kornblith, S.J., Greenwald, D.P., Javan, C.D., y Madonia, M.J. (1986). Family psychoeducation, social skills training and maintenance chemotherapy in the after care treatment of schizophrenia. 1: Oneyear effects of a controlled study on relapse and expressed emotion. Archives of General Psychiatry, 43, 633-642.

Hogarty, G., Macevoy, J.P., Munetz, M. et al. (1988). Dose of fluphenazine, familial expressed emotion, and outcome in schizophrenia. Results of a two-year controlled study. Archives of General Psychiatry, 45, 797-805.

Jarne, A., Costa, J.M., y Sanz, A. (1995). Trastornos cognitivos en la esquizofrenia. En Costa Molinari, JM. (Ed.) Trastornos cognitivos en psicopatología. Barcelona: JR Prous Editores.

John, C.H., y Dodgson, G. (1994). Inductive reasoning in delusional thought. Journal of Mental Health, 3, 31-49.

Johnson, D.A., Ludlow, J., Street, K., y Taylor, R.D. (1987). Double-blind comparision of half-dose and standar-dose flupenthixol decanoate in the maintenance treatment of stabilised outpatients with schizophrenia. British Journal of Psichiatry, 151, 634-638.

Lang, P.J., y Bus, A.H. (1965). Psychological deficit in schizophrenia: II. Interference and activation. Journal of Abnormal Psychology, 70, 77-106.

Leff, J., Kuipers, L., Berkowitz, R., Eberleinfries, R., y Sturgeon, D. (1982). A controlled trial of intervention in the families of schizophrenic families. British Journal of Psychiatry, 141, 121-134.

Leff, J., Kuipers, L., Berkowitz, R., y Sturgeon, D. (1985). A controlled trial of social intervention in the families of schizophrenic patients: Two- year follow- up. British Journal of Psychiatry, 146, 594-600.

Leff, J., Berkowitz, R., Shavit, A., Strachan, A., Glass, I., y Vaughn, C. (1989). A trial of family therapy $v$. a relatives group for schizophrenia. British Journal of Psychiatry, 154, 58-66.

Leff, J., Berkowitz, R., Shavit, A., Strachan, A., Glass, I., y Vaughn, C. (1990). A trial of family therapy $v$. a relatives group for schizophrenia: two- year follow- up. British Journal of Pschiatry, 157, 571-577.
Liberman, R.P. (1972). Behavior modification of schizophrenia: a review. National Institute of Mental Health Schizophrenia Bulletin, 6, 37-88.

Liberman, R.P. (1982). Tratamiento conductual del paciente crónico en el Hospital Mental. En H. Leitenberg (Comp.), Modificación y Terapia de Conducta, Vol I. Madrid, Ediciones Morata.

Lidde, PH. (1990). Prefrontal and subcortical disfuntion in schizophrenia. Handsbook of Schizophrenia. Amsterdam: Elsevier.

Lloyd, L.E., y Garlington, W.K. (1968). Weekly variations in performance on a token economy psychiatric ward. Behaviour Research And Therapy, 6, 407-410.

Margo, A., Hemsley, D.R., y Slade, P.D. (1981). The effects of varying auditory input on schizophrenic hallucinations. British Journal of Psychiatry, 139, 122-127.

Mintz, S. y Alpert, M. (1972). Imagery vividness, reality testing and schizophrenic hallucinations. Journal of Abnormal and Social Psychology, 19, 310-316.

Neufeld, R.W. (1991). Memory in paranoid schizophrenia. En P.A. Magaro (Comp.), Annual review of Psychopathology, 1, 231261.

Nuechterlein, K.H., y Dawson, M.E. (1984). Information processing and attentional functioning in the developmental course of schizophrenic disorders. Schizophrenia Bulletin, 10, 160-203.

Nydegger, R.V. (1972). The elimination of hallucinatory and delusional behaviour by verbal conditioning and assertive training: a case study. Journal of Behavior Therapy and Experimental Psychiatry, 3, 225-227.

Oltmanns, T.F., y Maher, B.A. (Comps.) (1988). Delusional beliefs. Nueva York: Wiley.

Penn, D.L., y Mueser, K. (1996). Research update of psychosocial treatment of schizophrenia. American Journal of Psychiatry, 153, 3, 607-617.

Reybee, J., y Kinch, B. (1973).Treatment of auditory hallucinations using focusing. En P. Slade y R. Bentall (1988), Sensory deception. A scientific analysis of hallucination. London: Croom Helm.

Roder, V., Brenner, H.D., Hodel, B., y Kienzle, N. (1996). Terapia integrada de la esquizofrenia. Barcelona: Ariel. 
Ruiz Vargas, J.M. (1987). Esquizofrenia: un enfoque cognitivo. Madrid: Alianza.

Sanz, J., y Cañive, J. (1996). Psicoeducación de familiares de pacientes esquizofrénicos. En Aldaz y Vázquez (Comps.), Esquizofrenia: fundamentos psicológicos y psiquiatricos de la rehabilitación. Madrid: Siglo XXI.

Schaefer, H.H., y Martin, P.L. (1966). Behavioral therapy for "apathy" of hospitalized schizophrenics. Psychological Reports, 19, 1147-1158.

Shriqui, C.L., y Nasrallah, H.A. (Eds.) (1996). Aspectos actuales en el tratamiento de la esquizofrenia. Madrid: Edimusa.

Siegel, J.M. (1975). Successful systematic desensitization in a chronic schizophrenic patient. Journal of Behaviour Therapy and Experimental Psychiatry, 6, 345-346.

Slade, P.D. (1972). The effects of systematic desensitization on auditory hallucinations. Behaviour, Research and Therapy, 10, 85-91.

Slade, P.D. (1973). The psychological investigation and treatment of auditory hallucinations: a second case report. British Journal of Medical Psychology, 46, 293-296.

Slade, P.D., y Bentall, R.P. (1988). Sensory deception: a scientific analysis of hallucination. Londres: Croom-Helm.

Stahl, J.R., y Leitenberg, H. (1982). Tratamiento conductual del paciente crónico en el hospital mental. En H. Leitenberg, Modificación y terapia de conducta. Tomo I. Madrid: Ediciones Morata.

Steffy, R.A. (1993). Cognitive deficits in schizophrenia. En K. Dobson y P. Kendall (Comps.), Psychopatology and cognition. Nueva York: Academic.

Tarrier, N., Barrowclough, C., Vaughn, C., Bamrah, J.S., Porceddu, K., Watts, S., y Freeman, $H$. (1988). The community management of schizophrenia: a controlled trial of a behavioural intervention with families to reduce relapse. British Journal of Psychiatry, 153, 532-542.
Tarrier, N. (1989). The community management of schizophrenia: a two year followup of a beha:'ioural intervention with families. British Journal of Pschiatry, 154, 625628.

Tarrier, N. (1989). Electrodermal activity expressed emotion and out-come in schizophrenia. British Journal of Pysichiatry, 155, (5), 51-56.

Wagner, B.R. (1968). The training of attending and abstracting responses in chronic schizophrenics. Journal of Experimental Research in Personality, 3, 77-78.

Watts, F., y Clements, J. (1971). The modification of schizophrenic hallucinations and associated delusions: a case report. Unpublished paper.

Watts, F.N., Powell, E.G., y Austin, S.V. (1973). The modification of abnormal beliefs. British Journal of Medical Psychology, 46, 359-363.

Weingaertner, A.H. (1971). Self-administered aversive stimulation with hallucinating hospitalised schizophrenics. Journal of Consulting and Clinical Psychology, 36, 422-429.

Wincze, J.P., Leitenberg, H., y Agras, W.S. (1972). The effects of token reinforcement of the delusional verbal behaviour of chronic paranoid schizophrenics. Journal of Applied Behaviour Analysis, 5, 247-262.

Winkler, R.C. (1970). Management of chronic psychiatric patients by a token reinforcement system. Journal of Applied Behaviour Analysis, 3, 47-55.

Young, H.F., Bentall, R.P., Slade, P.D., y Dewey, M.E. (1987). The role of brief instructions and suggestibility in the elicitation of hallucinations in normal and psychiatric subjects. Journal of Nervous and Mental Disease, 175, 41-48.

Zubin, J., y Spring, B. (1977). Vulnerability: a new view of schizophrenia. Journal of Abnormal Psychology, 86, 103-126. 